\title{
Refractory pruritus from malignant cholestasis: management
}

\author{
GVMC Fernando ${ }^{1,2}$ Nancy Preston ${ }^{3}$ \\ ${ }^{1}$ National Centre for \\ Primary Care and Allergy Research, University of Sri Jayewardenepura, Nugegoda, \\ Sri Lanka \\ ${ }^{2}$ Department of Family \\ Medicine, University of Sri Jayewardenepura Faculty of Medical Sciences, Nugegoda, \\ Sri Lanka \\ ${ }^{3}$ International Observatory of End of Life Care, Lancaster University, Lancaster, UK
}

To cite: Fernando GVMC, Preston N. BMJ Supportive \& Palliative Care Epub ahead of print: [please include Day Month Year]. doi:10.1136/ bmjspcare-2019-002051

\section{Abstract}

This case report deals with a patient managed in a tertiary-care cancer hospital who suffered pruritus associated with malignant cholestasis. His symptoms were resistant to conventional treatment with ursodeoxycholic acid, chlorpheniramine and cholestyramine. Hence, the multifactorial origin of malignancy- associated pruritus was considered. Correctable factors were corrected and generally the treatment was aimed at possible aetiologies. There were barriers related to insufficient resources available for symptom palliation in this particular setting, which could potentially reduce optimum symptom control. However, various pharmacotherapies and non-pharmacological measures which could potentially have helped relieve pruritus are described and future scope for research in this area discussed.

\section{Background}

'Pruritus' or 'itch' is a sensory percep- tion which leads to the reflexive act of scratching. It is designated as a 'nocicep- tive' perception since pruritus likely arises from exogenous (eg, caustic substances) or endogenous (eg, histamine) noxious stimuli which are weaker in intensity than those which generate pain. ${ }^{1}$ In cholestasis, interruption of enterohepatic bile acid circulation results in sequestration underneath the skin, an endogenous source of pruritogenic stimulation.

Generally, pruritus is not a common cancer symptom. ${ }^{2}$ It is part of the Memo- rial Symptom Assessment Scale. ${ }^{3}$ Pruritus is more commonly associated with specific cancers such as Hodgkin's lymphoma, leukaemia and myeloma. ${ }^{45}$ In $10 \%$ of instances, it is a paraneoplastic manifes- tation of malignancy. ${ }^{4}$ Pruritus may occur from the malignancy itself, resulting in cholestasis, renal failure from treat- ments like adverse reactions to opioids or targeted anti-cancer therapies. ${ }^{67}$

A quarter of those with cholestasis experience distressing pruritus. ${ }^{8}$ Common

bile duct stenting is considered the gold- standard treatment associated in malignant cholestasis. In conservative management, ursodeoxycholic acid (stimulates hepa- tobiliary secretions) is recommended. ${ }^{5}$ Some evidence support the selective serotonin reuptake inhibitor (SSRI) antidepressant sertraline as a safe, effective first-line therapy. ${ }^{10}$ Rifampicin can help, but the risk of hepatotoxicity, which could potentially worsen cholestasis, limits its use. ${ }^{11}$ The opioid-receptor antagonists butorphanol and naltrexone are not suit- able for those on opioids for symptom relief. ${ }^{12} 13$ Dronabinol, interferon-alpha, mirtazapine, phenobarbital, propofol, thalidomide and ursodeoxycholic acid are some other options. ${ }^{1}$ 


\section{Case presentation}

The patient was a sexagenarian who suffered from progressive dysphagia and significant weight loss for over 2 months before he presented to the hospital. He was diagnosed with advanced adenocarci- noma (stage T4b) in the lower third of the oesophagus, encroaching on the stomach. It was deemed unresectable. The tumour had metastasised to the lower lumbar distant nodes, liver and lumbar vertebrae (including the porta hepatis).

He received chemoradiation to palliate his dysphagia. Two weeks following the completion of radiotherapy, food intake had improved, with a weight gain of $4 \mathrm{~kg}$ over the following month. He also suffered from backache, treated with ibuprofen plus omeprazole with satisfac- tory results.

Two months later, he was re-admitted with worsening anorexia, backache, jaun- dice, generalised pruritus and weakness. He was referred to the palliative care unit (PCU), where the following management strategies were employed. He had to be discharged after 2 days and managed as an outpatient due to bed shortage.

\section{Case assessment}

Investigations demonstrated cholestatic jaundice (total bilirubin of $80 \mu \mathrm{mol} / \mathrm{L}$ ) and elevated serum bile salt levels $(32 \mu \mathrm{mol} / \mathrm{L})$ which were attributed to the porta- hepatis lymphadenopathy. Marginal hypercalcaemia (serum ionised calcium of $2.85 \mathrm{mmol} / \mathrm{L}$ ) and anaemia (haemoglobin=9 $\mathrm{g} / \mathrm{dL}$ ) were the identifiable causes for the constitutional symptoms like asthenia and anorexia (hypercalcaemia may also cause pruritus). ${ }^{8}$

Renal function was stable making uraemia a remote cause for pruritus. Possible iatrogenic causes for pruritus were ibuprofen and morphine (the latter was introduced at the initial visit).

\section{Case management}

He was started on ursodeoxycholic acid on his initial visit. This is the first-line agent, with multiple mecha- nisms of action in cholestasis-related pruritus. ${ }^{89}$ This was combined with the oral antihistamine chlorphe- niramine. This was a good choice for two reasons: wide availability in the hospital and as a first- generation (sedating) antihistamine proven supe- rior to second-generation drugs (eg, loratadine) for pruritus. ${ }^{14}$ Cholestyramine was added due to inade- quate response in the follow-up visit 2 weeks later. Notwithstanding cholestyramine's ability to bind avidly with bile salts thus eliminating them from skin, there is evidence cholestyramine is often ineffective since the blood level of bile salts correlates poorly with clinical pruritus. ${ }^{15} 16$

The single definitive treatment of proven universal efficacy for pruritus from cholestasis is biliary drainage. ${ }^{8}$ Despite minimally invasive procedures such as endo- scopic ultrasonography or endoscopic retrograde chol- angiopancreatography, in our clinical setting with limited resources, patients with cancer do not have access to such sophisticated procedures, and further, the patient stressed his strong aversion towards invasive procedures.

Four pints of blood were transfused and meticulous rehydration done with intravenous $0.9 \%$ saline. Intra- venous bisphosphonate (etidronate) was administered as a single dose $7.5 \mathrm{mg} / \mathrm{kg}$ along with highdose dexa- methasone (24mg daily in four divided doses), which improved hypercalcaemia, and possibly the related constitutional symptoms and pruritus. ${ }^{17}$ Etidronate also potentially contributed towards the relief of back pain associated with vertebral metastasis. ${ }^{117}$

Ibuprofen, a non-steroidal anti-inflammatory drug (NSAID), may cause allergy and hence pruritus. ${ }^{18}$ However, he tolerated the drug well over the previous 2 months. 
Oral morphine at the PCU visit was titrated to anal- gesic effect, and this had rendered him free of back pain by the second review without worsening pruritus. ${ }^{19}$ Since his pain was controlled with oral morphine, it was not prudent to try naltrexone for pruritus since it could exaggerate pain.

\section{Case Outcome}

At his second review 2 weeks later in the palliative care clinic, the back pain was still controlled. Fatigue had improved significantly, and his appetite returned. He was unconcerned by the yellowish discolouration of his skin and mucous membranes. The itching continued. He had been discharged from the inpatient facility by the oncology team without notifying the palliative team.

\section{Discussion}

The 5-hydroxytryptamine ( $\left.5-\mathrm{HT}_{3}\right)$ receptor antagonist ondansetron, which is of proven efficacy in alleviating pruritus due to cholestasis and sertraline (SSRI), would have been potential options. Parenteral lignocaine is effective for intractable cholestatic pruritus and is a widely available local anaesthetic. ${ }^{1}$ Other pharmaco- logical agents are efficacious to varying degrees. Incon- veniences in generalised itching has limited the use of bland emollients and levomenthol cream which have also been found to be effective. Non-pharmacological measures like acupuncture, plasma exchange, transcutaneous electrical nerve stimulation (TENS) and ultravi- olet-B combined with crotamiton have shown variable promise. ${ }^{16}$

\section{Conclusions}

Malignant cholestasis-associated pruritus can be a trou- blesome symptom. Therapeutic use of ursodeoxycholic acid combined with antihistamines may fail to relieve the symptom sufficiently. Even though cholestyramine in the standard management of cholestatic pruritus is common in this clinical setting, it is less predictable. Pruritus in malignancy may also be complicated by factors like NSAIDs, opioids or metabolic derangements such as uraemia and hypercalcaemia, which also need to be addressed.

A growing knowledge base on alternative pharma- cological and non-pharmacological treatments brings optimism about the future management of pruritus associated with malignant cholestasis. $5-\mathrm{HT}_{3}$ receptor antagonists, SSRIs, lignocaine, levomenthol, TENS, acupuncture, plasma exchange and UV+crotamiton are among these novel therapeutic modalities.

Twitter GVMC Fernando @chemetf and Nancy Preston @ nancypreston16

Acknowledgements This article is a modification of one of the assignments submitted by the principal author as part of his Postgraduate Diploma in Palliative Medicine at Cardiff University, UK.

Contributors GF involved himself with the clinical care of the patient, which subsequently led to a discussion with NP. NP guided GF's clinical management in the role of a supervisor. The case report prepared by GF was scrutinised by NP while making additions and modifications. Further, the final overall version submitted for publication is guaranteed for by GF.

Funding The authors have not declared a specific grant for this research from any funding agency in the public, commercial or not-for-profit sectors.

Competing interests None declared.

Patient consent for publication Not required.

Provenance and peer review Not commissioned; internally peer reviewed.

\section{ORCID iD}

GVMC Fernando http://orcid.org/0000-0003-2689-7306 


\section{References}

1. 1 Pittelkow MR, Loprinzi CL, Pittelkow TP. Pruritus and sweating in palliative medicine-Oxford Medicine [Internet], 2018. Available: http://oxfordmedicine.com/view/10.1093/ med/9780199656097.001.0001/med-9780199656097chapter-112

2. 2 Deshields TL, Potter P, Olsen S, et al. The persistence of symptom burden: symptom experience and quality of life of cancer patients across one year. Support Care Cancer 2014;22:1089-96.

3. 3 Chang VT, Hwang SS, Feuerman M, et al. The Memorial Symptom Assessment Scale Short Form (MSAS-SF). Cancer 2000;89:1162-71.

4. 4 McFarland DC, Shaffer KM, Polizzi H, et al. Associations of physical and psychologic symptom burden in patients with Philadelphia chromosome-negative myeloproliferative neoplasms. Psychosomatics 2018;59:472-80.

5. 5 Butler DF. Pruritus and systemic disease: background, pathophysiology, epidemiology [Internet]. Available: https:// emedicine.medscape.com/article/1098029-overview\#a6 [Accessed 11 Jun 2018].

6. 6 Katcher J, Walsh D. Opioid-induced itching: morphine sulfate and hydromorphone hydrochloride. J Pain Symptom Manage 1999;17:70-2.

7. 7 Ensslin CJ, Rosen AC, Wu S, et al. Pruritus in patients treated with targeted cancer therapies: systematic review and meta- analysis. J Am Acad Dermatol 2013;69:708-20.

8. Bassari R, Koea JB. Jaundice associated pruritis: a review of pathophysiology and treatment. World J Gastroenterol 2015;21:1404-13.

9. 9 Beuers U. Drug insight: mechanisms and sites of action of ursodeoxycholic acid in cholestasis. Nat Clin Pract Gastroenterol Hepatol 2006;3:318-28.

10. 10 Mayo MJ, Handem I, Saldana S, et al. Sertraline as a first-line treatment for cholestatic pruritus. Hepatology 2007;45:666-74.

11. 11 Howard P, Twycross R, Grove G, et al. Rifampin (INN rifampicin).J Pain Symptom Manage 2015;50:891-5.

12. 12 Dawn AG, Yosipovitch G. Butorphanol for treatment of intractable pruritus. J Am Acad Dermatol 2006;54:527-31.

13. 13 Twycross RG. Introducing palliative care. Radcliffe Publishing, 2003: $210 \mathrm{p}$.

14. 14 Simons FER, Simons KJ. H1 antihistamines: current status and future directions. World Allergy Organ J 2008;1:145-55.

15. 15 Herndon JH. Pathophysiology of pruritus associated with elevated bile acid levels in serum. Arch Intern Med 1972;130:632-7.

16. 16 Seccareccia D, Gebara N. Pruritus in palliative care: getting up to scratch. Can Fam Physician 2011;57:1010-3.

17. 17 Singer FR, Ritch PS, Lad TE, et al. Treatment of hypercalcemia of malignancy with intravenous etidronate. A controlled, multicenter study. The Hypercalcemia Study Group. Arch Intern Med 1991;151:471-6.

18. 18 Roujeau JC. Clinical aspects of skin reactions to NSAIDs. Scand J Rheumatol Suppl 1987;65:131-4.

19. 19 Mansour-Ghanaei F, Taheri A, Froutan H, et al. Effect of oral naltrexone on pruritus in cholestatic patients. World J Gastroenterol 2006;12:1125-8. 